\title{
Role of Immune Therapies for Myeloma
}

\author{
Jacalyn Rosenblatt, MD, and David Avigan, MD
}

\begin{abstract}
Immune therapy has emerged as a promising area of cancer therapeutics based on its potential for tumor selectivity and targeting of chemotherapy-resistant clones. Allogeneic transplantation produces durable remissions in a subset of patients, albeit at the cost of graftversus-host disease. Recent years have witnessed efforts to induce more selective immune responses via dendritic cell vaccines, autologous and engineered T-cell therapy, and immune checkpoint blockade. Optimizing these immunotherapeutic approaches, understanding how to best use them in combination, and determining how to integrate them with standard anti-myeloma therapy could provide the potential to alter the natural history of this disease. (J Natl Compr Canc Netw 2015;13:1440-1447
\end{abstract}

\section{NCCN: Continuing Education}

\section{Accreditation Statement}

This activity has been designed to meet the educational needs of physicians and nurses involved in the management of patients with cancer. There is no fee for this article. No commercial support was received for this article. The National Comprehensive Cancer Network (NCCN) is accredited by the ACCME to provide continuing medical education for physicians.

NCCN designates this journal-based CME activity for a maximum of 1.0 AMA PRA Category 1 Credit $^{\mathrm{TM}}$. Physicians should claim only the credit commensurate with the extent of their participation in the activity.

NCCN is accredited as a provider of continuing nursing education by the American Nurses Credentialing Center's Commission on Accreditation.

NCCN designates the education activity for a maximum of 1.0 contact hour. Accreditation as a provider refers to recognition of educational activities only; accredited status does not imply endorse-

From the Section of Hematological Malignancies and Bone Marrow Transplantation, Beth Israel Deaconess Medical Center, Boston, Massachusetts.

Submitted June 17, 2015; accepted for publication October 21, 2015. Jacalyn Rosenblatt, MD, has disclosed that she receives Grant/Research Support from Millennium Pharmaceuticals, Inc. and Bristol-Myers Squibb, and is a Scientific Advisor for Janssen Pharmaceutica. David Avigan, MD, has disclosed that he receives Grant/Research Support from Genus Oncology LLC and Astex Pharmaceuticals and is a Scientific Advisor for Celgene Corporation, Synta Pharmaceuticals Corp, and Bristol-Myers Squibb.

Correspondence: Jacalyn Rosenblatt, MD, Section of Hematological Malignancies and Bone Marrow Transplantation, Beth Israel Deaconess Medical Center, KS121, Boston, MA 02215. E-mail: jrosenb1@bidmc harvard.edu

David Avigan, MD, Section of Hematological Malignancies and Bone Marrow Transplantation, Beth Israel Deaconess Medical Center, KS121, Boston, MA 02215. E-mail: davigan@bidmc.harvard.edu ment by NCCN or ANCC of any commercial products discussed/ displayed in conjunction with the educational activity. Kristina M. Gregory, RN, MSN, OCN, is our nurse planner for this educational activity.

All clinicians completing this activity will be issued a certificate of participation. To participate in this journal CE activity: 1) review the learning objectives and author disclosures; 2 ) study the education content; 3) take the posttest with a $66 \%$ minimum passing score and complete the evaluation at http://education.nccn.org/ node/76117; and 4) view/print certificate.

Release date: November 11, 2015; Expiration date: November 11, 2016

\section{Learning Objectives}

Upon completion of this activity, participants will be able to:

- Describe the rationale for use of immunotherapy in treating multiple myeloma

- List the immunotherapeutic strategies currently being evaluated in clinical trials for treatment of multiple myeloma

\section{EDITOR}

Kerrin M. Green, MA, Assistant Managing Editor, JNCCN-Journal of the National Comprehensive Cancer Network

Ms. Green has disclosed that she has no relevant financial relationships.

\section{CE AUTHORS}

Deborah J. Moonan, RN, BSN, Director, Continuing Education Ms. Moonan has disclosed that she has no relevant financial relationships.

Ann Gianola, MA, Senior Manager, Continuing Education Accreditation and Program Operations

Ms. Gianola has disclosed that she has no relevant financial relationships. Kristina M. Gregory, RN, MSN, OCN, Vice President, Clinical Information Operations

Ms. Gregory has disclosed that she has no relevant financial relationships. Rashmi Kumar, PhD, Senior Manager, Clinical Content

Dr. Kumar has disclosed that she has no relevant financial relationships. 


\section{Allogeneic Transplantation: Proof of Principle for Immunotherapy}

Outcomes for patients with multiple myeloma have dramatically improved since the introduction of novel agents, such as proteasome inhibitors and immunomodulatory dugs. Nonetheless, for most patients, current treatment options are not curative, and most patients will ultimately experience relapse with refractory disease. The unique efficacy of cellular therapy is highlighted by the observation that allogeneic transplantation results in durable remissions in a subset of patients. Data from the European registry demonstrated an overall survival of $28 \%$ at 7 years following myeloablative allogeneic transplantation. ${ }^{1}$

However, regimen-related toxicity caused by myeloablative conditioning; the prolonged period of immune dysregulation, which increases increasing the susceptibility for opportunistic infections; and the lack of specificity of alloreactive lymphocytes, contributing to graft-versus-host disease, result in prohibitive treatment-associated morbidity and mortality. Reduced-intensity conditioning regimens have lowered some of these risks, but at the cost of increased risk for relapse. ${ }^{2,3}$ Tandem autologous followed by nonmyeloablative transplantation has been studied in an effort to segregate the toxicity and maximize the therapeutic synergy of doseintensive chemotherapy and immune-mediated targeting of malignant plasma cells. In fact, a series of phase II studies evaluating sequential autologous and nonmyeloablative transplantation showed encouraging results. ${ }^{4,5}$ However, randomized controlled trials evaluating tandem autologous transplantation versus sequential autologous/nonmyeloablative allogeneic transplantation have shown mixed results. ${ }^{6-12}$ The largest study, conducted by the Blood and Marrow Transplant Clinical Trials Network (BMT CTN), did not demonstrate a progression-free survival (PFS) or overall survival (OS) advantage in the allogeneic transplantation arm. ${ }^{10}$ Consistent with this result, 2 meta-analyses of published trials showed that treatment-associated mortality was higher in patients undergoing nonmyeloablative transplantation, resulting in no difference in PFS or OS, despite higher complete response (CR) rates in the allogeneic transplant group. ${ }^{13,14}$ It is notable, however, that the 2 studies with the longest follow-up did demonstrate a survival advantage for patients undergoing autologous followed by nonmyeloablative allogeneic transplantation. ${ }^{11,12}$ The role of allogeneic transplantation continues to be explored, particularly as part of primary therapy for patients with poor-risk features. Alternatively, the development of immunotherapy that can harness the unique benefit of immune-mediated plasma cell killing, while sparing the toxicity associated with allogeneic transplantation, has the potential to improve outcomes for patients with multiple myeloma.

\section{Immune Dysregulation in Multiple Myeloma}

Myeloma is characterized by deficiencies in cellular and humoral immunity that contribute to the immunosuppressive milieu promoting disease progression and immune escape. ${ }^{15}$ Patients with myeloma exhibit impairment in humoral immunity, with a resultant increased risk of bacterial infection. Alterations in natural killer (NK) cell biology result in decreased NK cell capacity to lyse malignant plasma cells. T-cell immunity is compromised because of the lack of effective antigen presentation, polarization toward an inhibitory phenotype, and increased levels of cytokines that suppress function. The complex interaction between stromal elements, such as regulatory $\mathrm{T}$ cells, myeloid-derived suppressor cells, and plasmacytoid dendritic cells (DCs), contributes to an immunosuppressive microenvironment that promotes tumor growth. Negative costimulatory molecules, including CTLA4 and PD-L1, play a vital role in promoting $\mathrm{T}$-cell exhaustion and anergy in the setting of malignancy. Upregulation of the PD-1/ PD-L1 pathway has been noted in patients with myeloma. ${ }^{16,17}$ Plasmacytoid DCs have also been shown to express PD-L1, invoking their role in modulating T-cell exhaustion and immune suppression. ${ }^{18}$ The development of immune therapy for myeloma focuses on the development of strategies that reverse critical aspects of the immunosuppressive milieu in the context of activation of myeloma-specific immunity (Figure 1).

\section{Vaccine Approaches to Stimulate Myeloma-Specific Immunity}

Myeloma-associated antigens have been identified that are selectively expressed by the malignant clone. An ideal candidate would be uniformly expressed by 


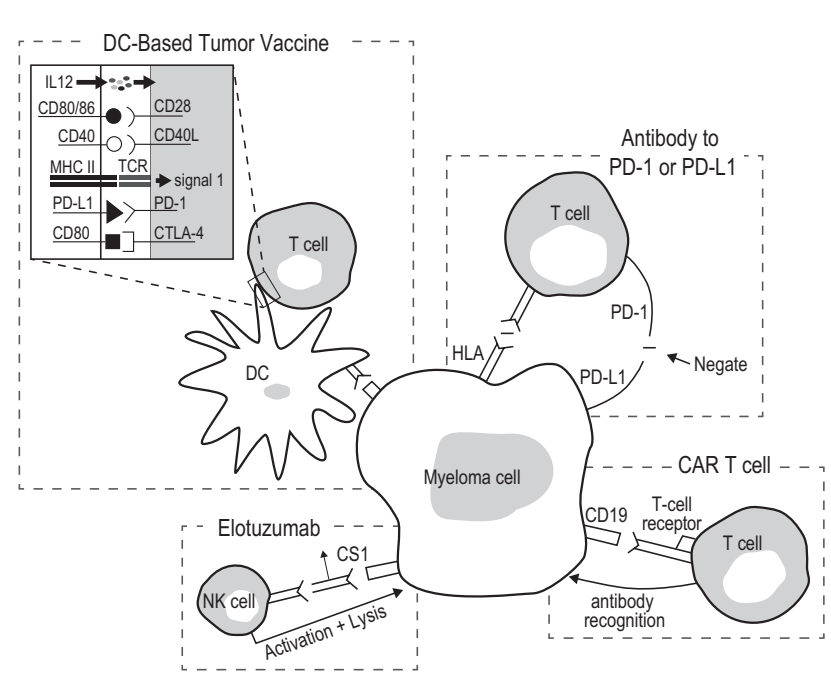

Figure 1 Targets of immune-based therapy for multiple myeloma. Abbreviations: CAR, chimeric antigen receptor; DC, dendritic cell; MHC, major histocompatibility complex; NK, natural killer; TCR, T-cell receptor.

the malignant clone; play an important role in the biology of the malignant cell, such that downregulation in the setting of immunologic pressure would not be readily seen; be immunogenic; and be highly specific to the malignant clone, limiting off-target toxicity. The idiotype protein is highly specific to the malignant plasma cell clone as the product of the unique VDJ rearrangement in this population, but its immunogenicity is uncertain. ${ }^{19,20}$ Cancer-testis antigens such as NY-ESO are minimally expressed in normal tissues, highly expressed by myeloma cells, and upregulated with disease evolution. ${ }^{21}$ Other examples of immunostimulatory myeloma-associated peptides that have been explored both preclinically and in clinical trials include WT-1, RHAMM, HSP96, MUC1, MAGE, HM1.24, and DKK1.22-25

Myeloma cells present tumor-associated antigens in the absence of necessary costimulation, resulting in ineffective effector immune responses. As such, developing a potent myeloma vaccine relies on effectively presenting myeloma-associated antigens in the context of necessary costimulation, in order to evoke activated effector cell responses. In contrast to malignant plasma cells, DCs are potent antigenpresenting cells that strongly express costimulatory molecules and secrete stimulatory cytokines, which results in T-cell activation. In an effort to evoke effective myeloma-associated immunity, strategies to load tumor antigens onto DCs have been evaluated. In one of the first clinical trials to evaluate DC-based vaccination in the setting of multiple myeloma, 12 patients were vaccinated with idiotype-pulsed DCs following autologous transplantation. Of these patients, 2 demonstrated idiotype-specific T-cell responses and sustained clinical remission. ${ }^{26}$ Although this early study showed promise, subsequent clinical trials evaluating idiotype-pulsed DC vaccines in patients in multiple myeloma elicited immune responses but generally lacked clinical efficacy. ${ }^{27-29}$ APC8020, a product generated by pulsing autologous DCs with autologous serum containing idiotype protein, was studied in patients with multiple myeloma following autologous transplant in a single-arm phase II clinical trial. Compared with historical controls, no difference in PFS was observed; however, a significant improvement in OS was demonstrated. ${ }^{30}$ The observation of improved OS despite a lack of difference in PFS is consistent with observations relating to immunotherapy in the setting of solid tumors, wherein a survival advantage has been demonstrated despite a lack of disease regression. ${ }^{31}$ This highlights the potential for immune modulation to effect response to subsequent therapy, and the need for careful consideration of methods for measuring the clinical impact of immunotherapy.

Although the idiotype protein was the first myeloma-associated antigen to be studied in clinical trials, idiotype has been shown to be a weak immunogen, increasing interest in targeting other, more strongly immunogenic myeloma-associated antigens. Peptidebased vaccines against WT1 and RHAMM-R3 have been studied in clinical trials. WT1 peptide vaccine was administered in the setting of refractory disease. An immune response was evoked in response to vaccination, and evidence was seen of clinical activity, with minimal clinical response according to European Group for Blood and Marrow Transplantation (EGBMT) criteria. ${ }^{32}$

In 2 phase I/II clinical trials evaluating an RHAMM peptide vaccine in patients with acute myeloid leukemia, myelodysplastic syndromes, and multiple myeloma, a total of 7 patients with multiple myeloma were treated with RHAMM peptide vaccine. Six of 7 patients with multiple myeloma treated on 1 of these 2 clinical trials demonstrated an immune response to vaccination, and in 3 of 7 patients with multiple myeloma, a clinical effect of vaccination was observed..$^{33,34}$ A recent phase I clinical trial demonstrated the safety and immunologic potency of vaccinating patients with DC pulsed with mRNA specific for MAGE-3, survivin, 
or B-cell maturation antigen (BCMA) after autologous transplant. An antigen-specific immune response was demonstrated in 2 of 12 patients. ${ }^{35}$ Clinical trials are ongoing to investigate vaccination against NY-ESO or MAGE-A3 and granulocyte macrophage colony-stimulating factor (GMCSF; ClinicalTrials.gov identifier: NCT00090493); MUC1 peptide and GM-CSF (ClinicalTrials.gov identifier: NCT01232712); and XBP1, CD138, and CS1 in patients with smoldering myeloma (ClinicalTrials.gov identifier: NCT01718899).

A limitation to vaccine approaches that target an individual tumor antigen is the potential for tumor cells to evade immune recognition through downregulating expression of that antigen. As such, whole-cell vaccine approaches have been studied, with the advantage of presenting patient-specific, and potentially unidentified, antigens to immune effector cells. Strategies to introduce whole tumor cell antigens include pulsing with lysates, ${ }^{36}$ electroporation with tumor-derived RNA or DNA, ${ }^{37-39}$ and loading of tumor-derived apoptotic bodies. ${ }^{40}$

Our group developed a vaccine model in which patient-derived myeloma cells are fused with autologous ex vivo-generated DCs, such that a broad array of myeloma-associated antigens, including neoantigens arising from unique mutations of a given patient, are presented in the context of DC-mediated costimulation. A phase I clinical trial evaluating the DC/ myeloma fusion vaccine was completed in patients with advanced multiple myeloma. ${ }^{41}$ Vaccination resulted in the expansion of myeloma-specific $T$ cells in most patients, despite a median of 4 prior regimens in the treated patients; $66 \%$ of patients demonstrated disease stabilization, ranging from 2 months to greater than 2 years. In a subsequent phase II clinical study, vaccination with $\mathrm{DC} /$ myeloma fusions was administered after autologous transplantation. ${ }^{42}$ The period of early posttransplant lymphopoietic reconstitution was associated with the suppression of regulatory $T$ cells and the expansion of myeloma-reactive $\mathrm{T}$ cells. This environment offered a potent platform for vaccination, resulting in further boosting of the myelomadirected immune response.

Notably, vaccination was associated with the delayed conversion of partial to complete responses in a subset of patients, suggesting the targeting of posttransplant residual disease. ${ }^{42}$ Vaccination was well tolerated and, importantly, was not associated with clinically significant cytopenias or autoimmunity. The most common adverse event related to vaccination involves transient erythema and tenderness at the vaccine site. In preclinical studies, we demonstrated that lenalidomide augments immune response to vaccination. ${ }^{43}$ Given the evolving role of lenalidomide maintenance after autologous transplantation and its potential role as a immune modulatory agent in the posttransplant setting, we initiated a national randomized phase II through the Clinical Trials Network (CTN protocol 1401) in which patients will be randomized to receive the fusion vaccine and lenalidomide maintenance compared with lenalidomide maintenance alone after autologous transplantation. The primary end point is to evaluate the CR rate at 1 year after transplant. As a secondary end point, an integrated assessment of humoral and cellular immunity and correlation with clinical impact will be explored.

\section{Adoptive T-Cell Therapy}

Vaccine therapy is limited by poor responsiveness of native effector cell populations, particularly in patients with more advanced disease. An alternative strategy is the ex vivo generation of activated T-cell populations that may show greater responsiveness to help to restore myeloma-specific immunity and be engineered to specifically target myeloma-associated antigens. An initial clinical trial evaluated adoptively transferred ex vivo activated and expanded $\mathrm{T}$ cells administered to patients with multiple myeloma after autologous transplantation in an effort to improve immune reconstitution, augment antimyeloma immunity, and amplify response to vaccination. A total of 42 patients were randomized to receive ex vivo anti-CD3/anti-CD28 costimulated autologous $\mathrm{T}$ cells on day 12 or 100 after autologous transplant, and to receive 2 doses of pneumococcal vaccine with or without an initial vaccination prior to T-cell collection. Notably, this study demonstrated that a priming vaccination prior to T-cell collection was critical for the development of antipneumococcal immunity. ${ }^{44}$ In a subsequent study, 54 patients received ex vivo costimulated T cells on day 2 after autologous transplant. Patients who were HLA2positive received a pneumococcal vaccine plus a tumor vaccine composed of HLA2.1-restricted peptides telomerase and survivin, whereas HLA2.1negative patients received only a pneumococcal 
vaccine. Immune response to vaccination was $\mathrm{ob}$ served in both groups, without a difference in eventfree survival..$^{45} \mathrm{~A}$ phase II clinical trial is underway evaluating activated $\mathrm{T}$ cells administered in combination with a peptide vaccine against MAGE-3 (ClinicalTrials.gov identifier: NCT01245673). In another ongoing study, activated $\mathrm{T}$ cells are administered in conjunction with an idiotype vaccine after autologous transplantation (ClinicalTrials.gov identifier: NCT01426828).

A potentially promising approach of obtaining myeloma-specific T cells for ex vivo manipulation and adoptive transfer involves the use of bone marrowinfiltrating cells (MILs). ${ }^{46}$ These cells are thought to represent a polyclonal population of $\mathrm{T}$ cells reactive to myeloma-associated antigens but subject to tumorinduced anergy in the bone marrow microenvironment. In preclinical studies, bone marrow-derived lymphocytes have been shown to demonstrate greater proliferative response to ex vivo activation and expansion and greater myeloma-specific cytotoxicity compared with lymphocytes isolated from the peripheral blood. ${ }^{47}$ A clinical trial is being conducted in which MILs undergo ex vivo activation and are reinfused on day 3 after autologous transplantation (ClinicalTrials. gov identifier: NCT00566098).

\section{Chimeric Antigen Receptor T-Cell Therapy}

Although adoptive transfer of ex vivo-expanded autologous $\mathrm{T}$ cells shows promise, challenges remain in isolating and expanding myeloma-reactive $\mathrm{T}$ cells from the blood or bone marrow. An exciting alternative approach lies in the generation of genetically modified chimeric antigen receptor cells (CARs). CAR T cells are synthetically engineered via transduction of a specific variable fragment of a monoclonal antibody into the T-cell receptor. Similar to native $\mathrm{T}$ cells, CARs function via activation of the zeta-chain of the CD3 complex, with second- and third-generation CARs containing additional costimulation, such as 4-1BB, CD28, or OX40, providing a constitutive source of $\mathrm{T}$-cell activation and bypassing the complexity of the signaling between antigen-presenting cells and T cells. CD19 CARs have been extensively studied in chronic lymphocytic leukemia and acute lymphoblastic leukemia, ${ }^{48}$ with particularly exciting durable remissions demonstrated in patients with refractory ALL.
CAR T-cell therapy has been associated with significant toxicity, such as cytokine-release syndrome and neurotoxicity. CD19 CARs are being evaluated in multiple myeloma in an effort to target the CD19. positive myeloma-initiating cell population. A recent publication reported on the first patient treated in a clinical trial in which CD19-specific CAR T cells were administered after high-dose melphalan. ${ }^{49}$ Remarkably, a complete response was observed in this patient with relapsed myeloma, who had previously been treated with 9 regimens, including autologous transplantation. Notably, at the time of publication, response was ongoing at 12 months. Several other clinical trials evaluating CARs in multiple myeloma are underway, including a phase I study of a CD28-based CAR directed at the kappa light chain (ClinicalTrials.gov identifier: NCT00881920) and a CAR directed against CD138 (CART-138; ClinicalTrials.gov identifier: NCT01886976). Other targets, including the NKG2D receptor, whose ligands are expressed on myeloma cells and enhance NK cell-mediated lysis, have demonstrated therapeutic potency in mouse models, ${ }^{50}$ and will be studied in upcoming clinical trials.

\section{NK Cell Therapy}

NK cells represent an important immune effector cell population that is not reliant on HLA restriction and has the potential for potent antimyeloma activity. NK cells undergo activation as a result of either increased expression of activating ligands, or decreased expression of inhibitory ligands. IPH2101, a human monoclonal antibody against common inhibitory KIRs (killer cell immunoglobulin-like receptors), enhances NK cell-mediated killing of autologous myeloma cells. The drug was studied in a phase I trial in patients with relapsed/refractory multiple myeloma, resulting in disease stabilization in a subset of patients, without evidence of disease regression. ${ }^{51}$ IPH2101 is currently being evaluated in patients with smouldering myeloma (ClinicalTrials. gov identifiers: NCT0124855 and NCT01222286), and in combination with lenalidomide in patients with relapsed disease (ClinicalTrials.gov identifier: NCT01217203).

Elotuzumab (HuLuc63), a humanized anti-CS1 monoclonal antibody (mAb) that can augment $\mathrm{NK}$ cell-mediated antimyeloma immunity, induces my- 
eloma cell death through antibody-dependent cellular cytotoxicity. In a phase I study of patients with relapsed/refractory myeloma, elotuzumab demonstrated minimal single-agent activity. ${ }^{52}$ In preclinical studies, it demonstrated synergy with bortezomib and lenalidomide. In a phase I study of elotuzumab and bortezomib, an overall response rate of $48 \%$ was observed. ${ }^{53}$ In a phase II study of elotuzumab in combination with lenalidomide and dexamethasone, the overall response rate was $84 \%(n=71) .{ }^{54}$ In a recent phase III randomized controlled trial, elotuzumab plus lenalidomide and dexamethasone was compared with lenalidomide and dexamethasone alone in patients with relapsed/refractory multiple myeloma. Notably, the combination of elotuzumab, lenalidomide, and dexamethasone resulted in a 30\% reduction in the risk of death or disease progression compared with the control arm. ${ }^{55}$ Adverse events related to elotuzumab have included lymphopenia and infusion reactions, such as fevers and hypertension. ${ }^{55}$ An alternative strategy has been the development of NK CARs, in which human NK cells are genetically engineered to express a CAR that is specific to a myeloma associated target, in addition to a costimulatory signaling domain. In preclinical studies, NK CARs targeting CS1 potently lysed human myeloma cells in a xenograft model. ${ }^{56}$

\section{Checkpoint Blockade}

The PD-L1/PD-1 pathway is a negative costimulatory pathway that induces a state of T-cell exhaustion, preventing activation and expansion of T-cell populations. A greater understanding of the critical role that this pathway plays in mediating tumor tolerance has led to clinical trials evaluating antibodies to both PD-1 and PD-L1, in which durable clinical responses were observed in patients with advanced malignancy. ${ }^{57}$ Adverse events related to immune checkpoint blockade have included autoimmune complications, including pneumonitis and colitis, which are treated with steroids. Preclinical studies have supported the role that this pathway plays in blunting immune-mediating killing of plasma cells in multiple myeloma. ${ }^{16}$

Clinical trials are underway to investigate antibodies to PD-1 and PD-L1 in hematologic malignancies, including multiple myeloma. It is important to consider that, to date, the hematologic malignancy in which blockade of the PD-L1/PD-1 pathway has shown efficacy is Hodgkin disease, ${ }^{57}$ which is characterized by a significant native immune infiltrate in the tumor bed. Optimizing response to PD-L1/ PD-1 blockade in multiple myeloma may require combining this approach with strategies that stimulate myeloma-reactive $\mathrm{T}$ cells in the blood and bone marrow. In preclinical studies, we have shown that PD-1 blockade augments immune response to DC/ myeloma fusion cell vaccination. ${ }^{16}$ In an effort to couple immune checkpoint blockade with strategies that expand myeloma-reactive $T$ cells, we are conducting a clinical trial in which patients with multiple myeloma are treated with pidilizumab in combination with $\mathrm{DC} /$ myeloma fusion cell vaccination in the early period after autologous transplantation (ClinicalTrials.gov identifier: NCT01067287).

\section{Conclusions}

Immunotherapy has the potential to improve outcomes for patients with multiple myeloma. DC vaccines, adoptive and genetically engineered $\mathrm{T}$-cell therapy, monoclonal antibodies, and immune checkpoint blockade have each demonstrated encouraging results. Future directions lie in understanding how to incorporate each of these approaches with standard antimyeloma therapy, optimizing the timing of immunotherapy, and learning how to combine immunotherapeutic strategies to maximize potency and limit toxicity. The field of immunotherapy is poised to alter the natural history of multiple myeloma, and change it from a chronic to a potentially curable disease.

\section{References}

1. Gahrton G, Tura S, Ljungman P, et al. Prognostic factors in allogeneic bone marrow transplantation for multiple myeloma. J Clin Oncol 1995;13:1312-1322.

2. Shah N, Callander N, Ganguly S, et al. Hematopoietic stem cell transplantation for multiple myeloma: guidelines from the American Society for Blood and Marrow Transplantation. Biol Blood Marrow Transplant 2015;21:1155-1166.

3. Bensinger W. Allogeneic stem cell transplantation for multiple myeloma. Hematol Oncol Clin North Am 2014;28:891-902.

4. Kroger N, Perez-Simon JA, Myint H, et al. Relapse to prior autograft and chronic graft-versus-host disease are the strongest prognostic factors for outcome of melphalan/fludarabine-based dose-reduced allogeneic stem cell transplantation in patients with multiple myeloma. Biol Blood Marrow Transplant 2004;10;698-708.

5. Mohty M, Boiron JM, Damaj G, et al. Graft-versus-myeloma effect following antithymocyte globulin-based reduced intensity conditioning allogeneic stem cell transplantation. Bone Marrow Transplant 2004;34:7784. 
6. Garban F, Attal M, Michallet M, et al. Prospective comparison of autologous stem cell transplantation followed by dose-reduced allograft (IFM99-03 trial) with tandem autologous stem cell transplantation (IFM99-04 trial) in high-risk de novo multiple myeloma. Blood 2006;107:3474-3480.

7. Bruno B, Rotta M, Patriarca F, et al. A comparison of allografting with autografting for newly diagnosed myeloma. N Engl J Med 2007;356:1110 1120 .

8. Giaccone L, Storer B, Patriarca F, et al. Long-term follow-up of a comparison of nonmyeloablative allografting with autografting for newly diagnosed myeloma. Blood 2011;117;6721-6727.

9. Rosinol L, Perez-Simon JA, Sureda A, et al. A prospective PETHEMA study of tandem autologous transplantation versus autograft followed by reduced-intensity conditioning allogeneic transplantation in newly diagnosed multiple myeloma. Blood 2008;112;3591-3593.

10. Krishnan A, Pasquini MC, Logan B, et al. Autologous haemopoietic stemcell transplantation followed by allogeneic or autologous haemopoietic stem-cell transplantation in patients with multiple myeloma (BMT CTN 0102): a phase 3 biological assignment trial. Lancet Oncol 2011;12:11951203.

11. Bjorkstrand B, Iacobelli S, Hegenbart U, et al. Tandem autologous/ reduced-intensity conditioning allogeneic stem-cell transplantation versus autologous transplantation in myeloma: long-term follow-up. J Clin Oncol 2011;29:3016-3022.

12. Gahrton G, Iacobelli S, Bjorkstrand B, et al. Autologous/reduced-intensity allogeneic stem cell transplantation vs autologous transplantation in multiple myeloma: long-term results of the EBMT-NMAM2000 study. Blood 2013;121:5055-5063.

13. Armeson KE, Hill EG, Costa LJ. Tandem autologous vs autologous plus reduced intensity allogeneic transplantation in the upfront management of multiple myeloma: meta-analysis of trials with biological assignment. Bone Marrow Transplant 2013;48:562-567.

14. Kharfan-Dabaja MA, Hamadani M, Reljic T, et al. Comparative efficacy of tandem autologous versus autologous followed by allogeneic hematopoietic cell transplantation in patients with newly diagnosed multiple myeloma: a systematic review and meta-analysis of randomized controlled trials. J Hematol Oncol 2013;6:2.

15. Romano A. Conticello C, Cavalli M, et al. Immunological dysregulation in multiple myeloma microenvironment. Biomed Res Int 2014;2014:198539.

16. Rosenblatt J, Glotzbecker B, Mills H, et al. PD-1 blockade by CT-011, antiPD-1 antibody, enhances ex vivo T-cell responses to autologous dendritic cell/myeloma fusion vaccine. J Immunother 2011;34:409-418.

17. Hallett WH, Jing W, Drobyski WR, Johnson BD. Immunosuppressive effects of multiple myeloma are overcome by PD-L1 blockade. Biol Blood Marrow Transplant 2011;17:1133-1145.

18. Ray A, Das DS, Song Y, et al. Targeting PD1-PDL1 immune checkpoint in plasmacytoid dendritic cell interactions with T cells, natural killer cells and multiple myeloma cells. Leukemia 2015;29:1441-1444.

19. King CA, Spellerberg MB, Zhu D, et al. DNA vaccines with single-chain Fv fused to fragment $C$ of tetanus toxin induce protective immunity against lymphoma and myeloma. Nat Med 1998;4:1281-1286.

20. Campbell MJ, Esserman L, Byars NE, et al. Idiotype vaccination against murine B cell lymphoma. Humoral and cellular requirements for the full expression of antitumor immunity. J Immunol 1990;145:1029-1036.

21. Pellat-Deceunynck $C$, Mellerin MP, Labarriere N, et al. The cancer germline genes MAGE-1, MAGE-3 and PRAME are commonly expressed by human myeloma cells. Eur J Immunol 2000;30:803-809.

22. Takahashi T, Makiguchi Y, Hinoda Y, et al. Expression of MUC1 on myeloma cells and induction of HLA-unrestricted CTL against MUC1 from a multiple myeloma patient. J Immunol 1994;153:2102-2109.

23. Yasukawa $M$, Fujiwara $H$, Ochi $T$, et al. Clinical efficacy of WT1 peptide vaccination in patients with acute myelogenous leukemia and myelodysplastic syndrome. Am J Hematol 2009;84:314-315.

24. Atanackovic D, Arfsten J, Cao Y, et al. Cancer-testis antigens are commonly expressed in multiple myeloma and induce systemic immunity following allogeneic stem cell transplantation. Blood 2007;109:1103-1112.

25. Bae J, Munshi NC, Anderson KC. Immunotherapy strategies in multiple myeloma. Hematol Oncol Clin North Am 2014;28:927-943.

26. Reichardt VL, Milazzo C, BruggerW, et al. Idiotype vaccination of multiple myeloma patients using monocyte-derived dendritic cells. Haematologica 2003;88:1139-1149.

27. Yi Q, Szmania S, Freeman J, et al. Optimizing dendritic cell-based immunotherapy in multiple myeloma: intranodal injections of idiotypepulsed CD40 ligand-matured vaccines led to induction of type-1 and cytotoxic T-cell immune responses in patients. Br J Haematol 2010;150:554-564.

28. Bendandi M, Rodriguez-Calvillo M, Inoges $S$, et al. Combined vaccination with idiotype-pulsed allogeneic dendritic cells and soluble protein idiotype for multiple myeloma patients relapsing after reduced-intensity conditioning allogeneic stem cell transplantation. Leuk Lymphoma 2006;47:29-37.

29. Roeven MW, Hobo W, Schaap N, Dolstra H. Immunotherapeutic approaches to treat multiple myeloma. Hum Vaccin Immunother 2014;10:896-910.

30. Lacy MQ, Mandrekar S, Dispenzieri A, et al. Idiotype-pulsed antigenpresenting cells following autologous transplantation for multiple myeloma may be associated with prolonged survival. Am J Hematol 2009;84:799_ 802 .

31. Kantoff PW, Schuetz TJ, Blumenstein BA, et al. Overall survival analysis of a phase II randomized controlled trial of a Poxviral-based PSA-targeted immunotherapy in metastatic castration-resistant prostate cancer. J Clin Oncol 2010;28:1099-1105.

32. Tsuboi A, Oka Y, Nakajima H, et al. Wilms tumor gene WT1 peptide-based immunotherapy induced a minimal response in a patient with advanced therapy-resistant multiple myeloma. Int J Hematol 2007;86:414-417.

33. Greiner J, Schmitt A, Giannopoulos K, et al. High-dose RHAMM-R3 peptide vaccination for patients with acute myeloid leukemia, myelodysplastic syndrome and multiple myeloma. Haematologica 2010;95:1191-1197.

34. Schmitt M, Schmitt A, Rojewski MT, et al. RHAMM-R3 peptide vaccination in patients with acute myeloid leukemia, myelodysplastic syndrome, and multiple myeloma elicits immunologic and clinical responses. Blood 2008;111:1357-1365.

35. Hobo W, Strobbe L, Maas F, et al. Immunogenicity of dendritic cells pulsed with MAGE3, Survivin and B-cell maturation antigen mRNA for vaccination of multiple myeloma patients. Cancer Immunol Immunother 2013;62:1381-1392.

36. Lee JJ, Choi BH, Kang HK, et al. Induction of multiple myeloma-specific cytotoxic T lymphocyte stimulation by dendritic cell pulsing with purified and optimized myeloma cell lysates. Leuk Lymphoma 2007;48:2022-2031.

37. Liu J, Hamrouni A, Wolowiec D, et al. Plasma cells from multiple myeloma patients express B7-H1 (PD-L1) and increase expression after stimulation with IFN-\{gamma\} and TLR ligands via a MyD88-, TRAF6-, and MEK dependent pathway. Blood 2007;110:296-304.

38. Signori E, Iurescia S, Massi E, et al. DNA vaccination strategies for antitumour effective gene therapy protocols. Cancer Immunol Immunother 2010;59:1583-1591.

39. Fioretti D, Iurescia S, Fazio VM, Rinaldi M. DNA vaccines: developing new strategies against cancer. J Biomed Biotechnol 2010;2010:174378.

40. Spisek R, Chevallier P, Morineau N, et al. Induction of leukemia-specific cytotoxic response by cross-presentation of late-apoptotic leukemic blasts by autologous dendritic cells of nonleukemic origin. Cancer Res 2002;62:2861-2868.

41. Rosenblatt J, Vasir B, Uhl L, et al. Vaccination with dendritic cell/tumor fusion cells results in cellular and humoral antitumor immune responses in patients with multiple myeloma. Blood 2011;117:393-402.

42. Rosenblatt J, Avivi I, Vasir B, et al. Vaccination with dendritic cell/tumor fusions following autologous stem cell transplant induces immunologic and clinical responses in multiple myeloma patients. Clin Cancer Res 2013;19:3640-3648.

43. Luptakova K, Rosenblatt J, Glotzbecker B, et al. Lenalidomide enhances anti-myeloma cellular immunity. Cancer Immunol Immunother 2013;62:39-49.

44. Rapoport AP, Stadtmauer EA, Aqui N, et al. Restoration of immunity in lymphopenic individuals with cancer by vaccination and adoptive T-cell transfer. Nat Med 2005;11:1230-1237.

45. Rapoport AP, Aqui NA, Stadtmauer EA, et al. Combination immunotherapy using adoptive T-cell transfer and tumor antigen vaccination on the basis of hTERT and survivin after ASCT for myeloma. Blood 2011;117;788-797.

46. Garfall AL, Vogl DT, Weiss BM, Stadtmauer EA. Cellular immunotherapy for plasma cell myeloma. Bone Marrow Transplant 2013;48:1377-1386.

47. Noonan K, Matsui W, Serafini P, et al. Activated marrow-infiltrating lymphocytes effectively target plasma cells and their clonogenic precursors. Cancer Res 2005;65:2026-2034.

48. Grupp SA, Kalos M, Barrett D, et al. Chimeric antigen receptor-modified T cells for acute lymphoid leukemia. N Engl J Med 2013;368:1509-1518. 
Role of Immune Therapy in Myeloma

49. Garfall AL, Maus MV, Hwang WT, et al. Chimeric antigen receptor T cells against CD19 for multiple myeloma. N Engl J Med 2015;373:1040-1047.

50. Barber A, Meehan KR, Sentman CL. Treatment of multiple myeloma with adoptively transferred chimeric NKG2D receptor-expressing T cells. Gene Ther 2011;18:509-516.

51. Benson DM Jr, Bakan CE, Zhang S, et al. IPH2101, a novel anti-inhibitory KIR antibody, and lenalidomide combine to enhance the natural killer cell versus multiple myeloma effect. Blood 2011;118:6387-6391.

52. Zonder JA, Mohrbacher AF, Singhal S, et al. A phase 1, multicenter open-label, dose escalation study of elotuzumab in patients with advanced multiple myeloma. Blood 2012;120:552-559.

53. Jakubowiak AJ, Benson DM, Bensinger W, et al. Phase I trial of antiCS1 monoclonal antibody elotuzumab in combination with bortezomib in the treatment of relapsed/refractory multiple myeloma. J Clin Oncol 2012;30:1960-1965.
54. Lonial S, Vij R, Harousseau JL, et al. Elotuzumab in combination with lenalidomide and low-dose dexamethasone in relapsed or refractory multiple myeloma. J Clin Oncol 2012;30:1953-1959.

55. Lonial S, Dimopoulos M, Palumbo A, et al. Elotuzumab therapy for relapsed or refractory multiple myeloma. N Engl J Med 2015;373:621-631.

56. Chu J, Deng Y, Benson DM, et al. CS1-specific chimeric antigen receptor (CAR)-engineered natural killer cells enhance in vitro and in vivo antitumor activity against human multiple myeloma. Leukemia 2014;28:917-927.

57. Ansell SM, Lesokhin AM, Borrello I, et al. PD-1 blockade with nivolumab in relapsed or refractory Hodgkin's lymphoma. N Engl J Med 2015;372:311-319

\section{Instructions for Completion}

To participate in this journal CE activity: 1) review the learning objectives and author disclosures; 2 ) study the education content; 3 ) take the posttest with a $66 \%$ minimum passing score and complete the evaluation at http://education.nccn.org/ node/76117; and 4) view/print certificate. After reading the article, you should be able to answer the following multiple- choice questions. Credit cannot be obtained for tests completed on paper. You must be a registered user on NCCN.org. If you are not registered on NCCN.org, click on "New Member? Sign up here" link on the left hand side of the Web site to register. Only one answer is correct for each question. Once you successfully answer all posttest questions you will be able to view and/or print your certificate. Software requirements: Internet

\section{Posttest Questions}

1. True or False: Myeloma is characterized by deficiencies in cellular and humoral immunity that contribute to the immunosuppressive milieu promoting disease progression and immune escape.

2. The immunotherapeutic strategies currently being evaluated in clinical trials for treatment of multiple myeloma include:
a. Dendritic cell vaccines
b. Autologous and engineered T-cell therapy
c. Natural killer cell
d. immune checkpoint blockade
e. All the above
f. a, c, and d

3. Which of the following is true about elotuzumab?

a. It is a vaccine generated by pulsing autologous dendritic cells with autologous serum containing idiotype protein

b. In a recent phase III trial, the combination of elotuzumab, lenalidomide, and dexamethasone resulted in a reduction in the risk of death or disease progression compared with lenalidomide and dexamethasone

c. Both $a$ and b 